\title{
Invariant Manifold Reductions for Markovian Ion Channel Dynamics
}

\author{
James P. Keener \\ Department of Mathematics, \\ University of Utah, Salt Lake City, UT 84112
}

June 4, 2008

\begin{abstract}
We show that many Markov models of ion channel kinetics have globally attracting stable invariant manifolds, even when the Markov process is time dependent. The primary implication of this is that, since the dimension of the invariant manifold is often substantially smaller than the full master equation system, simulations of ion channel kinetics can be substantially simplified, with no approximation. We show that this applies to certain models of potassium channels, sodium channels, ryanodine receptors and $\mathrm{IP}_{3}$ receptors. We also use this to show that the original Hodgkin-Huxley formulations of potassium channel conductance and sodium channel conductance are the exact solutions of full Markov models for these channels.
\end{abstract}

Keywords: Markov models, ion channel kinetics, invariant manifold reduction.

\section{Introduction}

Ion channels are multiconformational proteins that open or close in a stochastic fashion, reflecting stochastic transitions between conformations. One popular way to model the dynamics of these transitions is as Markov jump processes. These Markov models are then used in conductance based models to study the dynamics of (for example) electrical activity in nerve cells, cardiac cells, muscle cells, etc.

As Markovian models have become more detailed and complicated, the need for ways to reduce this complexity has also become apparent. One often used technique to reduce model complexity is fast equilibrium or quasi-steady state analysis, in which the fastest transitions are taken to be in quasi-equilibrium. The reduced model then follows the dynamics on a slow manifold, which is of lower dimension than the full system.

A different reduction is possible if the Markovian process has a stable invariant manifold. If there is such a stable invariant manifold then after transients have decayed (which, we argue below, is 
always the case), the dynamics of the system are restricted to the stable invariant manifold, which being of lower dimension than the full system, is therefore simpler to simulate.

Several years ago (Chapter 3, [5]), it was noted that the Hodgkin-Huxley models for potassium and sodium conductances were actually the invariant manifolds of higher dimensional Markov channel models. At that time, no general explanation of why this is the case or when this might be applicable for other channel models or other Markov processes was provided. Furthermore, the global stability of this manifold was asserted, but no rigorous proof was provided.

The purpose of this paper is to provide that general explanation and in doing so, show that there are large classes of time-dependent Markovian jump processes that possess stable invariant manifolds. We then use this to reduce several well-known ion channels models. Specifically, we show reductions of models for potassium channels, sodium channels, and ryanodine receptors (RYR) and $\mathrm{IP}_{3}$ receptors. In addition, we show how the Hodgkin-Huxley formulations of potassium and sodium channel kinetics fit into this general framework, and in so doing reiterate that they are, in fact, exact solutions of full Markov models.

\section{Invariant Manifolds for Markovian Jump Processes}

We suppose that $X$ is a discrete random variable that can take a finite number of integer values $j=0,1, \cdots, N$. We also suppose that the probability of jumping from state $i$ to state $j$ in the time interval between $t$ and $t+d t$ is $k_{i j}(t) d t$, in the limit that $d t$ is small. Notice that this process need not be time independent. In particular, for ion channels, which is the application we have in mind, transition rates are often dependent on other time-varying variables such as voltage or ion concentrations. Finally, we suppose that the reaction kinetics are irreducible, meaning that there is no random variable $Y$ which takes on a subset of the values of $X$, which also forms a closed set.

The master equations for this stochastic process are well known to be given by [2]

$$
\frac{d p_{j}}{d t}=\sum_{i \neq j} k_{i j}(t) p_{i}-\sum_{i \neq j} k_{j i}(t) p_{j}, \quad j=0,1,2, \cdots
$$

where $p_{j}(t)$ is the probability of being in state $j$ at time $t$. Of necessity, $p_{j}(t) \geq 0$ and $\sum_{j} p_{j}(t)=1$. We can write the system (1) in matrix form

$$
\frac{d P}{d t}=A(t) P
$$

recognizing that $A=\left(a_{i j}\right)$ where

$$
a_{i j}=k_{j i}(t), i \neq j, \quad a_{i i}=-\sum_{j \neq i} k_{i j}(t) .
$$

We also write the conservation condition $\sum_{j} p_{j}(t)=1$ as $\hat{1}^{T} P=1$, where $\hat{1}^{T}=(1,1, \cdots, 1)$.

Any two solutions of the master equations have the same ultimate fate. That is, if $P_{1}(t)$ and $P_{2}(t)$ are two solutions of the master equations, then $Y=P_{1}(t)-P_{2}(t) \rightarrow 0$ as $t \rightarrow \infty$. More 
specifically, the $l^{1}$ norm of $Y,\|Y\|_{1}=\sum_{j}\left|y_{j}\right|$ is a monotone decreasing function of time [1]. This is not particularly interesting for time-independent processes, since all solutions approach the unique steady state, however, it is a much more substantial statement for time-dependent processes.

An invariant manifold for this equation is a vector, say $Q=\left(Q_{i}\right), \sum Q_{i}=1$, parameterized by the variable $q$, having the feature that $P=Q(q)$ is a solution of the differential equation (2) provided that $q$ satisfies a differential equation of lower dimension

$$
\frac{d q}{d t}=F(q, t) \text {. }
$$

It is easy to see that a sufficient condition for there to be an invariant manifold is that

$$
A(t) Q(q)=\sum_{j} \frac{\partial Q}{\partial q_{j}} F_{j}(q, t) .
$$

It follows immediately, that if the master equation has an invariant manifold it is globally stable. It also follows that the invariant manifold of lowest dimension is unique.

\section{Examples of Markov Processes with Invariant Manifolds}

The search for stable invariant manifolds is equivalent to the search for exact solutions of lower dimension. However, since the stable attractor is unique, we seek exact solutions that are of smallest possible dimension, rather than fully general solutions (for arbitrary initial data) as found in [4].

Several exact solutions of master equations are well known. In [2], it was shown that the master equation corresponding to the simple chemical reaction

$$
A \underset{\beta}{\stackrel{\alpha}{\rightleftarrows}} X
$$

with the concentration of species $A$ held fixed, has an exact solution which is Poisson distributed with parameter $q$, where $q$ is governed by the differential equation

$$
q_{t}=\alpha-\beta q .
$$

The proof of this given in [2] uses a generating function, and is therefore only valid if $\alpha$ and $\beta$ are constant. However, a straightforward calculation (by direct substitution) shows that the Poisson distribution is an exact solution even if $\alpha$ and $\beta$ are time dependent. Thus, the minimal stable attractor for the process (6) is the Poisson distribution parameterized by the parameter $q$.

Recently several more exact solutions have been found [4]. These include the multinomial distribution, the generalized Poisson distribution and the negative binomial distribution. Of these, the only one that is relevant for ion channel kinetics is the multinomial distribution.

Suppose the concentrations $C_{k}(t)$ of the chemical substances $S_{k}$ are governed by first order reaction kinetics, modeled by the deterministic differential equation

$$
\frac{d C}{d t}=A(t) C(t)
$$


where $C(t)=\left(C_{1}(t), C_{2}(t), \cdots, C_{n}(t)\right)^{T}$. Because this is a closed chemical reaction system, the off-diagonal entries $a_{i, j}$ are the first order reaction rates at which species $j$ is converted to species $i$. The diagonal entries $a_{i, i}$ are the rates at which species $i$ is converted to some other species, $a_{i, i}=-\sum_{j} a_{j, i}$. Thus, the form of this system is exactly the same as a master equation (2).

For a stochastic interpretation of this deterministic system, we let $X=\left(X_{1}(t), X_{2}(t), \cdots, X_{n}(t)\right)^{T}$ be a discrete random variable where $X_{k}(t)$ is the number of molecules in state $k$ at time $t$, and use the kinetic rates $a_{i, j}$ to generate a master equation for the probability that there are $i_{k}$ molecules of species $k$ at time $t$. In particular, the rate at which one molecule of species $k$ is converted to one molecule of species $j$ is $i_{k} a_{j, k}$.

A direct calculation (see [4]) verifies that the multinomial distribution

$$
p_{i_{1}, i_{2}, \cdots, i_{n}}=\frac{N !}{i_{1} ! i_{2} ! \cdots, i_{n} !} p_{1}^{i_{1}} p_{2}^{i_{2}} \cdots p_{n}^{i_{n}},
$$

is an exact solution of this master equation, provided the parameters of the distribution $P(t)=$ $\left(p_{1}(t), p_{2}(t), \cdots, p_{n}(t)\right)$ satisfy the differential equation (8).

The second useful feature of invariant manifolds is a multiplicative structure. That is, if $Q(q)$ is an invariant manifold for the stochastic process for the random variable $X$, and $R(r)$ is an invariant manifold for the stochastic process for the random variable $Y$, then the product $P=Q^{T} R$ is an invariant manifold for the random variable $Z=X \times Y$.

We can see how this works as follows. Suppose the random variable $Z$ has states that are denoted by two indices. Assume also that transitions are possible only between states for which one index, not two, differ. Thus, transitions between $S_{i, k}$ and $S_{j, k}$ or between $S_{i, k}$ and $S_{i, j}$ are permitted but no others. Further, we assume that the transition rates between $S_{i, k}$ and $S_{j, k}$ are independent of $k$, while the transition rates between states $S_{i, k}$ and $S_{i, j}$ are independent of $i$. We denote the transition rates between $S_{i, k}$ and $S_{j, k}$ by $\beta_{i, j}(t)$ and between $S_{i, k}$ and $S_{i, j}$ by $\gamma_{k, j}(t)$.

The master equation for this process is

$$
\frac{d p_{i, k}}{d t}=-\sum_{j} \beta_{i, j} p_{i, k}+\sum_{j} \beta_{j, i} p_{j, k}-\sum_{j} \gamma_{k, j} p_{i, k}+\sum_{j} \gamma_{j, k} p_{i, j},
$$

Now we let $Q$ be a solution of the lower dimensional master equation

$$
\frac{d Q_{i}}{d t}=-\sum_{j} \beta_{i, j} Q_{i}+\sum_{j} \beta_{j, i} Q_{j}
$$

and similarly let $S$ be the solution of the master equation

$$
\frac{d S_{k}}{d t}=-\sum_{j} \gamma_{k, j} S_{k}+\sum_{j} \gamma_{j, k} S_{j}
$$

It is an easy calculation to verify that $p_{i, k}=Q_{i} S_{k}$ is a solution of the master equation (10). 


\section{Ion channel kinetics with Invariant Manifolds}

We now use the above results to show that several well-known ion channels models have invariant manifolds of reduced dimension.

To do so we first note that while the above invariant manifolds were stated as applying to chemical reactions with chemical species $C$, the vector $C$ in (8) can equally well be viewed as a probability distribution function for any Markov process. Then, if there are multiple independent copies of this process, the random variable $X_{k}$ represents the number of elements that are in state $k$.

\subsection{Potassium Channels}

A simple model for the potassium channel found in the squid axon is that it is comprised of four independent subunits, each of which can be either open or closed, with voltage dependent transitions

$$
C \underset{\beta(V)}{\stackrel{\alpha(V)}{\rightleftarrows}} O
$$

The conducting state of the channel is that in which all subunits are open. If $k$ denotes the number of open subunits, then the state diagram is represented by

$$
S_{k} \underset{\beta(k+1)}{\stackrel{\alpha(N-k)}{\rightleftarrows}} S_{k+1} .
$$

This is a simple reaction network to which the above results apply. Specifically, since there are four independent copies of a two-state Markov process, the probability that $k$ of the subunits are in the open state is governed by the master equation

$$
\frac{d p_{k}}{d t}=(N-k-1) \alpha p_{k-1}+(k+1) \beta p_{k+1}-(N-k) p_{k}-k p_{k},
$$

with $N=4$. Furthermore, it follows from above (or a direct calculation) that the binomial distribution

$$
p_{k}(t)=\left(\begin{array}{c}
4 \\
k
\end{array}\right) q^{k}(1-q)^{4-k}
$$

is an invariant manifold for this master equation, provided the parameter $q$ satisfies the differential equation

$$
\frac{d q}{d t}=\alpha(V)(1-q)-\beta(V) q
$$

Furthermore the probability of being in the conducting state is $P_{O}=q^{4}$. This is exactly the open probability originally used by Hodgkin and Huxley [3], to model potassium channel conductance.

More recent data on $\mathrm{I}_{K s}$ potassium channels have suggested that the independent subunits may have multiple closed states. For example, in the model of Silva and Rudy [7] the subunit has three states,

$$
C_{2} \underset{\beta(V)}{\stackrel{\alpha(V)}{\rightleftarrows}} C_{1} \underset{\delta(V)}{\stackrel{\gamma(V)}{\rightleftarrows}} O
$$


where $\mathrm{C}_{1}$ and $\mathrm{C}_{2}$ are closed states and $\mathrm{O}$ is the open state. The conducting state of the channel is when all subunits are in the open state, and there are four independent subunits.

If $i_{C_{1}}, i_{C_{2}}$, and $i_{O}$ are the number of subunits in states $\mathrm{C}_{1}, \mathrm{C}_{2}$, and $\mathrm{O}$, respectively, then there are a total of fifteen different configurations for the four subunits. Thus, the master equations consist of fifteen differential equations. However, because the subunits are independent, there is a two-dimensional invariant manifold, given by the multinomial distribution

$$
p_{i_{C_{1}} i_{C_{2}} i_{O}}=\frac{4 !}{i_{C_{1}} i_{C_{2}} ! i_{O} !} p_{C_{1}}^{i_{C_{1}}} p_{C_{2}}^{i_{C_{2}}} p_{O}^{i_{O}},
$$

where the parameters $p_{C_{1}}, p_{C_{2}}$, and $p_{O}$, are governed by the differential equations

$$
\begin{aligned}
& \frac{d p_{C_{2}}}{d t}=\beta p_{C_{1}}-\alpha p_{C_{2}}, \\
& \frac{d p_{C_{1}}}{d t}=\alpha p_{C_{2}}+\delta p_{O}-(\beta+\gamma) p_{C_{1}},
\end{aligned}
$$

and $p_{C_{2}}+p_{C_{1}}+p_{O}=1$ for all time. Since the open state has $i_{O}=4$, the open probability is

$$
P_{O}=p_{O}^{4}
$$

but now the dynamics of $p_{O}$ are governed by two differential equations, rather than one, as in the Hodgkin-Huxley potassium channel model.

\subsection{Sodium Channels}

A simple model for the sodium channel of the squid axon is that it has three identical subunits that are activating (denoted by $m$ ) and one, also independent, that is inactivating (denoted by $h$ ). The transition probabilities are again voltage dependent, with

$$
C_{j} \underset{\beta_{j}(V)}{\stackrel{\alpha_{j}(V)}{\rightleftarrows}} O_{j}, j=m, h .
$$

It follows that both of these processes have invariant manifolds that are binomial distributions, with $N=3$ for $m$ and with $N=1$ for $h$. If we denote by $p_{j, k}$ the probability of $j$ open $m$ units and $k$ open $h$ units, (a total of eight possible states) then there is an invariant manifold for the master equations that is the product of these two binomial distributions,

$$
p_{j, k}=p_{j}^{3}(m) p_{k}^{1}(h),
$$

where by $p_{j}^{N}(a)$ is meant a binomial distribution for $N$ states with parameter $a$. The parameters $m$ and $h$ are governed by differential equations of the form

$$
\frac{d q}{d t}=\alpha_{q}(1-q)-\beta_{q} q, q=m, h .
$$

Furthermore, the probability of being in the conducting state is $p_{3,1}=m^{3} h$, which is also exactly the form originally used by Hodgkin and Huxley [3] to model sodium channel conductance. 


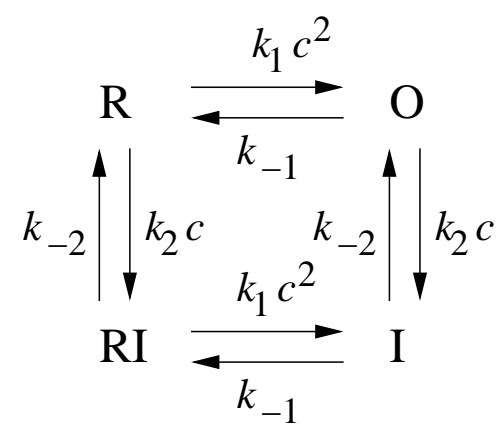

Figure 1: Model of the RyR due to Stern et al. (1999). R and RI are closed states, O is the open state, and I is the inactivated state.

\subsection{Ryanodine Receptors}

One of the earliest models of the ryanodine receptor, due to Stern et al.[8], is shown in Fig. 1. This is a four state model with closed states R and RI, inactivated state I, and open state O. Transitions between these states are calcium dependent, reflecting binding of calcium to binding sites. A modification of the Stern et al. model in which the rate constants $k_{1}$ and $k_{2}$ were assumed to be dependent on the concentration of calcium in the sarcoplasmic reticulum was used in [6].

The important observation here is that in these models the transition rates between $\mathrm{R}$ and $\mathrm{O}$ are identical to those between RI and I, and transition rates between $\mathrm{R}$ and RI are identical to those between $\mathrm{O}$ and $\mathrm{I}$. This can be viewed as the cross product of two two-state random variables. As discussed above, the master equations have a multiplicative stable invariant manifold of the form

$$
p_{\mathrm{R}}=p q, p_{\mathrm{RI}}=q(1-p), p_{\mathrm{O}}=q(1-p), p_{\mathrm{I}}=(1-p)(1-q),
$$

and the parameters $p$ and $q$ satisfy the differential equations

$$
\frac{d p}{d t}=k_{-1}(1-p)-k_{1} c^{2} p, \quad \frac{d q}{d t}=k_{-2}(1-q)-k_{2} c q
$$

\section{$4.4 \quad \mathrm{IP}_{3}$ Receptors}

The Keizer-DeYoung model for $\mathrm{IP}_{3}$ receptors [9] is shown in Fig. 2. This is an eight state model with states $S_{i j k}$ where $i, j$, and $k$ can take on values 0 or 1 . The index $i$ represents binding $(i=1)$ or unbinding $(i=0)$ of $\mathrm{IP}_{3}$, and the indices $j$ and $k$ represent binding or unbinding of calcium. In this model, the binding of calcium with index $j$ is activating, and the binding of calcium with index $k$ is inactivating, so that the only conducting state is $S_{110}$. In general, the concentration dependent transition rates are time dependent.

The observation that is relevant for this discussion is that there is a multiplicative structure, and 


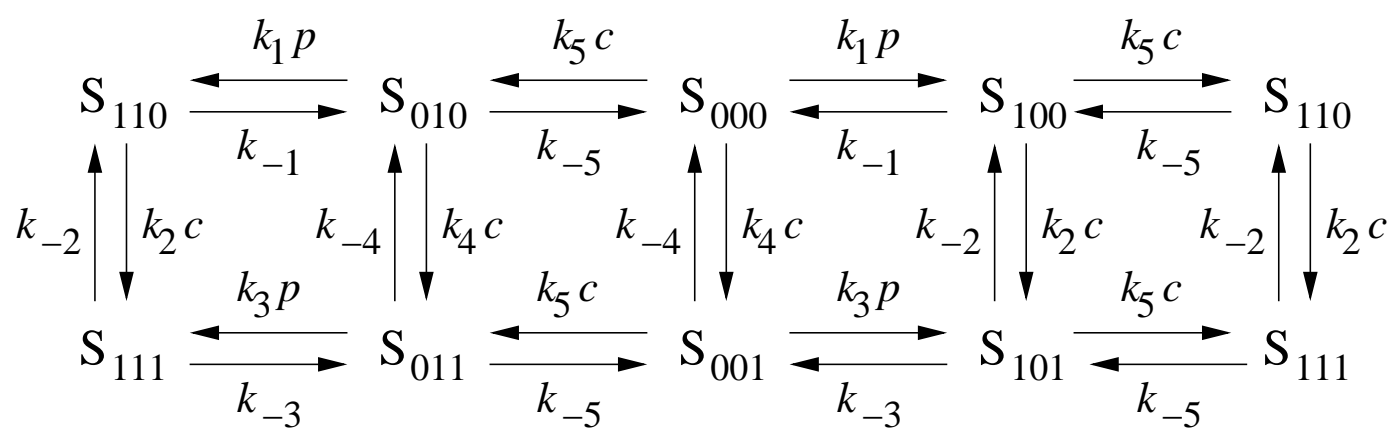

Figure 2: The binding diagram for the Keizer-DeYoung $\mathrm{IP}_{3}$ receptor model. Here, $c$ denotes Ca ${ }^{++}$, and $p$ denotes $\mathrm{IP}_{3}$.

hence an invariant manifold here. In particular, we let

$$
X=\left(\begin{array}{c}
000 \\
100 \\
101 \\
001
\end{array}\right), \quad Y=\left(\begin{array}{c}
010 \\
110 \\
111 \\
011
\end{array}\right)
$$

(that is, we let $X$ represent the states $S_{i 0 k}$, and let $Y$ represent the states $S_{i 1 k}$ ). Then the transitions within states $X$ and within states $Y$ are identical, and transitions between $X$ and $Y$ are the same for all the states. It follows that $P_{X}=q P_{Z}, P_{Y}=(1-q) P_{Z}$, is an invariant manifold for this process provided

$$
\frac{d P_{Z}}{d t}=A P_{Z}, \quad \frac{d q}{d t}=k_{-5}(1-q)-k_{5} c q,
$$

where $A$ is the matrix for the master equation for the states $X$ or $Y$. Thus, without approximation, the master equations can be reduced from a 7-dimensional to a 4-dimensional system.

\subsection{Multiple Channels}

Now suppose we wish to determine the number of open channels in a collection of $N$ independent channels. If the individual channel has $n$ states, then the distribution for the number of channels in each of the states is governed by the multinomial distribution (9). However, if only one of the states, say $j=1$ is a conducting state, then the probability distribution for the number of conducting states is the binomial distribution

$$
p_{i_{1}}(t)=\frac{N !}{i_{1} !\left(N-i_{1}\right) !} p_{1}^{i_{1}}\left(1-p_{1}\right)^{N-i_{1}},
$$

where $p_{1}(t)$ is the probability that a single channel is in the conducting state. It also follows that the expected value and variance of the number of conducting channels is

$$
E\left(i_{1}\right)=N p_{1}(t), \quad \operatorname{Var}=N p_{1}(t)\left(1-p_{1}(t)\right) .
$$

These formulas may appear to be rather unremarkable, since these are what one might expect from standard probability theory for independent, stationary Bernoulli trials. What makes this much 
more interesting, however, is that it is also correct for time dependent (non-stationary) Markov processes, once initial transients have decayed.

As a specific example, consider the sodium channels in the Hodgkin-Huxley model. This is an eight state model, however, as we saw above, the probability of being in the open state is $p_{1}(t)=m^{3} h$. The probability of having $i_{1}$ open sodium channels is given by (30) with $p_{1}(t)=m^{3} h$, where $m$ and $h$ satisfy the differential equations (25). Thus, the solution of the master equation for $N$ 8-state ion channels is fully described by a two dimensional manifold.

\section{Discussion}

We have shown that the master equations for ion channel models can have stable invariant manifolds that are of much lower dimension than the full system. The two features of these invariant manifolds that are most significant is that they are globally stable and that they are exact, even when the reaction kinetics are time dependent. These features are of somewhat limited value for typical chemical reaction networks since it is unusual for the rates of reaction to be time dependent, and initial data are often an important part of the problem specification. For time-independent processes, the smallest dimensioned invariant manifold is a point.

However, for ion channel kinetics these two features are quite useful. In contrast to chemical kinetics, for ion channels, the transition rates are typically time dependent and the initial data are not relevant. This is because for any realistic experimental procedure or physiological process, the initial data correspond to those channel states when the channel proteins were originally formed or when the cell was originally cultured, etc. By the time the experimental procedure is begun, (say, for example, a voltage clamp procedure on a neuron), the memory of the initial states is long gone. As a result, simulations intended to replicate experimental results need only use the invariant manifold description of channel kinetics. Of course, one must determine the initial position on the invariant manifold, but this is also easily (and correctly) done if the channels are held in a constant environment before the experiment is begun. This works because the time independent Markov process converges to a unique distribution and this becomes the appropriate initial distribution for the experiment.

Estimates of how long it take for initial transients to decay are possible. For example, for the potassium channel model (13), the smallest eigenvalue (in magnitude) of the matrix $A(t)$ is $\alpha(V)+\beta(V)$. For potassium channels, this is on the order of 1 /several milliseconds. Thus, transient effects decay less than a tenth of a second after the channel is inserted into the cellular membrane. More generally, if the process has been running for longer than the largest time constant of the underlying reaction network, transient effects have decayed.

We have also shown that the classical Hodgkin-Huxley formulations of potassium and sodium channel conductance are exact solutions of Markov models. This means that the solutions of the Hodgkin-Huxley equations and the solutions of a full Markov model with an 8-state sodium channel and a 4-state potassium channel model (after several milliseconds during which initial transients decay) are exactly the same, even though the first is a system of four differential equations and

the latter is a system of 13 differential equations. Similar reductions are possible for simulations of 
calcium dynamics using ryanodine receptors or $\mathrm{IP}_{3}$ receptors.

Acknowledgment: This research was supported in part by NSF Grants DMS-0211366 and DMS0718036 .

\section{References}

[1] B. Earnshaw and J. P. Keener. Global stability for time-dependent markov processes. to appear, 2008.

[2] C. W. Gardiner. Handbook of Stochastic Methods. Springer, New York, 2nd edition, 1985.

[3] A. L. Hodgkin and A. F. Huxley. A quantitative description of membrane current and its application to conduction and excitation in nerve. J. Physiol., 117:500-544, 1952.

[4] T. Jahnke and W. Huisinga. Solving the chemical master equation for monomlecular reaction systems analytically. J. Math. Biol., 54:1-26, 2007.

[5] J. P. Keener and J. Sneyd. Mathematical Physiology. Springer-Verlag, New York, 1998.

[6] T. R. Shannon, F. Wang, J. Puglisi, C. Weber, and D. M. Bers. A mathematical treatment of integrated Ca dynamics within the ventricular myocyte. Biophys. J., 87:3351-3371, 2004.

[7] J. Silva and Y. Rudy. Subunit interaction determines $\mathrm{I}_{K s}$ participation in cardiac repolarization and repolarization reserve. Circ., 121:1176-1182, 2005.

[8] M. D. Stern, L.-S. Song, H. Cheng, J. S.K. Sham, H. T. Yang, K. R. Boheler, and E. Rios. Local control models of cardiac excitation-contraction coupling: A possible role for allosteric interactions between ryanodine receptors. J. Gen. Phys., 113:469-489, March 1999.

[9] G. W. De Young and J. Keizer. A single pool [ $\left.\mathrm{IP}_{3}\right]$-receptor based model for agonist stimulated $\left[\mathrm{Ca}^{++}\right]$oscillations. Proc. Natl. Acad. Sci. USA, 89:9895-9899, 1992. 